\title{
PARP Inhibitors in Breast Cancer: Why, How, and When?
}

\author{
Chair: \\ Angelo Paradiso ${ }^{\mathrm{a}}$ \\ Participants: Eleni Andreopoulou ${ }^{b}$ Pierfranco Conte $^{c, d}$ Alexandru Eniue \\ Mahasti Saghatchian ${ }^{f}$

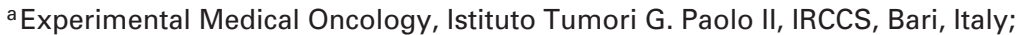 \\ bWeill Cornell Breast Center, Division of Hematology and Medical Oncology, Weill Cornell Medicine, New York, NY, USA; \\ 'Department of Surgery, Oncology and Gastroenterology, University of Padova, Padua, Italy; \\ dDivision of Medical Oncology 2, Istituto Oncologico Veneto IRCCS, Padua, Italy; \\ e Department of Breast Tumors, Cancer Institute 'Ion Chiricuta', Cluj-Napoca, Romania; \\ ${ }^{f}$ Breast Cancer Unit, American Hospital of Paris, Neuilly-sur-Seine, France
}

\section{Question 1: Do you think that we have the optimal drug companion test for PARP inhibitors (PARP-i) in our hands?}

Andreopoulou: Significant research is focusing on the development of companion diagnostic tests for PARP-i. Yet, patient selection with optimal drug companion product is limited. Earlier this year, the FDA approved the BRACAnalysis CDx test for use in identifying patients with breast cancer with deleterious or suspected deleterious germline BRCA mutation (gBRCAm) who may be eligible for olaparib. The effectiveness of the BRACAnalysis $\mathrm{CDx}$ test was established based on the OlympiAD trial population [1]. There is emerging evidence that a significant number of patients with BRCA1/BRCA2 wild-type (wt) breast cancer is also deficient in homologous recombination, and it is likely that these patients may derive a similar benefit from drugs targeting DNA repair pathways, necessitating the development of a companion diagnostic to identify these sporadic BRCA-like tumors. Earlier tests, such as homologous recombination deficiency (HRD) and others like it, have not been very successful in predicting response to PARP-i. In a recent report, HRDetect was demonstrated as a predictor of BRCA1 and BRCA2 deficiency based on mutational signatures associated with sensitivity to PARP-i [2]. Ultimately, whole-genome sequencing can support the implementation of precision medicine with PARP-i.

Conte: Today, we have good PARP-i and a good test, which is BRCA1/2 mutational status. There is room for improvement in terms of drug activity: i) not all BRCA-mutated (BRCAm) tumors respond to PARP-i; ii) some BRCAwt tumors can respond to PARP-i; iii) eventually all tumors initially responding will become PARP-i-resistant. The same applies for testing: i) the role of somatic BRCA mutation is still not fully elucidated; ii) the predictive role of HRD testing is still to be defined.

Eniu: Probably not. The relevance of somatic BRCA mutation in breast tumors is as yet unknown. Moreover, the mechanism of action of PARP-i is not directly linked to BRCA mutation, but rather takes advantage of the deficient homologous recombination repair mechanism in BRCA mutation carriers.

Saghatchian: Unfortunately, we have not yet identified the adequate companion test that would allow to predict response to PARP-i. PARP plays a major role in DNA repair at various levels, for single- and double-strand break repair.

As a consequence, PARP-i are of particular interest for patients with gBRCAm, for whom they induce synthetic lethality on tumor cells; however, patients with somatic mutations are also candidates for these types of treatments. So far, somatic mutations are detected in the context of specific trials through extensive molecular profiling of tumors; there is no diagnostic test looking at those mutations available for routine use. Moreover, patients with BRCAness profiles might also be candidates for such treatments, and again, such profiles are not detected by a ready-to-use companion test. Moreover, even in the presence of a BRCA mutation, tumor cells can resist PARP-i; thus, identification of a BRCA mutation does not predict potential resistance to PARP-i.

\section{KARGER}

() 2018 S. Karger GmbH, Freiburg

Fax +497614520714
Angelo Paradiso, MD 


\section{Question 2: What do you think is the right placement for PARP-i in the therapeutic strategy for the treatment of breast cancer today?}

Andreopoulou: Therapeutic strategy with PARP-i is essentially based on phase III clinical trial data that demonstrated a progression-free survival benefit in patients with gBRCAm, HER2-negative metastatic breast cancer.

The treatment indication includes patients with gBRCAm, HER2-negative metastatic breast cancer previously treated with chemotherapy. Hormone receptor-positive breast cancer should have been treated with prior endocrine therapy or be considered inappropriate for endocrine treatment.

Conte: This is not so easy to define based on actual data from trials. Data are more solid for triple-negative BRCAm tumors (first/second line of therapy), and they are a reasonable option for hormone receptor-positive BRCAm tumors (after several lines of endocrine therapy before switching to chemotherapy).

The efficacy in platinum-sensitive versus platinum-resistant patients is still unclear. There is not enough data on BRCAwt tumors.

Eniu: For the moment, the only approved indication of a PARP-i (olaparib) is for the treatment of patients with deleterious or suspected deleterious gBRCAm, HER2-negative metastatic breast cancer who have been treated with chemotherapy either in the neoadjuvant, or adjuvant, or metastatic setting. It can be speculated that future trials will promote the use of such agents from second/third line to first line. Ongoing studies are also evaluating its role in the adjuvant setting.

Saghatchian: Numerous phase II and phase III trials have been exploring the clinical activity of these drugs in various settings.

In locally advanced/metastatic breast cancer, the drugs have been tested in BRCAm triple-negative or not triple-negative, mainly pretreated patients; in most of the trials, patients were HER2-negative, platinum-resistant or not platinum-resistant, and/ or previously treated with anthracyclines and taxanes.

Mature data are only available for olaparib, with the OlympiA trial, showing benefit over standard therapy for gBRCAm metastatic breast cancer in a phase III trial; trial results with other PARP$i$ are eagerly awaited. Considering these data, olaparib should be proposed as single treatment to all patients with gBRCAm metastatic breast cancer progressing after anthracyclines and taxanes.

\section{Question 3: Should PARP-i or platinum-based chemotherapy regimens be administered in advanced disease?}

Andreopoulou: PARP-i and platinum salts have overlapping mechanisms of action and resistance mechanisms to some extent. It will be necessary to interpret PARP-i trial results in the context of ongoing trials of platinum therapy.
In the OlympiAD phase III study [1], patients who had prior platinum therapy as part of their treatment did not benefit, whereas those who did not have prior platinum treatment had more of a benefit. It is important to note the limitation for interpreting these results considering the small subsets of patients.

In the ABRAZO phase II study [3], notably in the group with prior platinum therapy, the longer interval from the prior platinum was associated with favorable outcome. Although there was no benefit for an interval of less than 2 months from treatment, there was a benefit for an interval of at least 6 months from treatment. A greater benefit was observed in the group that had not had prior platinum therapy.

In the EMBRACA phase III study [4], a superior clinical benefit was demonstrated in all subsets of patients, regardless of receptor subtype (hormone receptor-positive or triple-negative breast cancer), number of prior lines of chemotherapy, BRCA mutation type, and central nervous system metastasis.

It is recognized that BRCA-mutant cells are sensitized to the antineoplastic effects of DNA-damaging agents such as platinum. It was also recognized that sporadic triple-negative breast cancer often exhibited so-called BRCAness and sensitivity to platinumcontaining agents.

Conte: Please see my response above.

Eniu: We do not have data to reliably answer this question, as the patients enrolled in the standard arm of the registration trial received chemotherapy with capecitabine, eribulin, or vinorelbine, i.e. excluding platinum-based chemotherapy. Moreover, platinumresistant patients were excluded from this study. Consequently, we do not have direct comparison between PARP-i and platinum salts. On the other hand, the recently published TNT trial [5] reported that carboplatin achieved a $68 \%$ response rate in gBRCA1/2-mutated breast cancer in the first line, raising the important question regarding the relative benefit of PARP-i versus platinum regimens, also considering the important difference in the cost of PARP-i versus inexpensive platinum-based regimens.

Saghatchian: The mechanism of action of PARP-i is similar to what is observed with platinum-based chemotherapies. However, this question cannot be addressed in the absence of data allowing direct comparison of the two types of approaches at the clinical level.

Most of the trials have been comparing PARP-i to standard regimens of physician's choice (classically capecitabine, eribulin, gemcitabine, vinorelbine), excluding platinum-based chemotherapies, which had been used in previous lines of treatments for these patients.

Trials conducted with veliparib failed to show the benefit of the addition of the PARP-i to carboplatin-containing regimens (BROCADE trial [6]). For talazoparib, the ABRAZO [3] and EMBRACA [4] trials test the drug as single agent compared to various other regimes in patients resistant or not resistant to platinum agents. 


\section{Question 4: Should PARP-i only be applied in triple-negative breast cancer or in hormone receptor tumors, too?}

Andreopoulou: There is a role of PARP-i in breast cancer patients with BRCA1/2 germline mutations independently of the hormone receptor status.

Trial results were inconsistent in regards to the clinical benefit of PARP-i in hormone receptor-positive breast cancer.

The OlympiAD trial of the PARP-i olaparib compared one of three chemotherapy drugs, i.e. capecitabine, eribulin, or vinorelbine, with BRCA mutation carriers who had one or two prior treatments for metastatic breast cancer [1]. The study showed a significant progression-free benefit with olaparib confined to the triplenegative patients. Compared with the OlympiAD trial [1], in the ABRAZO trial [3] of the PARP-i talazoparib, there was a benefit in the estrogen receptor-positive patients as well. Because BRCA2 mutation carriers are more likely to have estrogen receptor-positive disease, this suggests that they can still benefit from PARP-i. Data from the EMBRACA trial [4] in patients with advanced HER2-negative breast cancer with gBRCAm had a significantly prolonged progression-free survival when treated with the PARP-i talazoparib; compared with those who received chemotherapy of physician's choice, talazoparib demonstrated superior clinical benefit in all subsets of patients, regardless of receptor subtype (hormone receptor-positive or triple-negative breast cancer), number of prior lines of chemotherapy, BRCA mutation type, and central nervous system metastasis.

Conte: Please see my answer above.

Eniu: Definitely, the selection criteria for PARP-i is the presence of gBRCAm, as this creates the environment of 'synthetic lethality' needed for PARP-i activity, irrespective of hormone receptor status. Therefore, PARP-i can be used both in triple-negative as well as in hormone receptor-positive patients carrying a gBRCAm.

Saghatchian: Although these drugs have been initially developed for triple-negative breast cancer patients (due to their high percentage among BRCAm patients and the lack of therapeutic options available for these patients), data from preclinical and clinical studies show the efficacy of the drugs in hormone receptor-positive and -negative patients with a BRCA mutation. For hormone receptor-positive patients, PARP-i show less efficacy than currently existing first-line treatment options (especially CDK4/6 inhibitors combined with hormonal treatments). Consequently, they are indicated in hormone receptor-positive patients, provided they have progressed after prior hormone receptor-positive targeted therapies.

The same approach might apply to HER2-positive patients, as explored in the ABRAZO trial (phase II trial of talazoparib) [3], where HER2-positive breast cancer was permitted, provided that the patient's disease was refractory to HER2-targeted therapy.

\section{Question 5: What could be the ideal clinical scenario for PARP-i utilization? Adjuvant, neoadjuvant, or advanced disease?}

Andreopoulou: In patients with early-stage BRCA-mutated breast cancer, we are focusing on the opportunity to cure with neoadjuvant/adjuvant therapy. Clinical trial testing of olaparib as adjuvant treatment in patients with gBRCAm high-risk HER2-negative primary breast cancer who have completed definitive local treatment and neoadjuvant or adjuvant chemotherapy (OlympiA) (ClinicalTrials.gov Identifier: NCT02032823) is underway. This trial will complete accrual this year and data are expected by 2020 . Combination therapy could potentially be the future success, the most exciting of which can be coupled immunotherapy.

In addition, there are a number of ongoing neoadjuvant and combination trials. Of interest, presented at ASCO 2018, a small feasibility study of neoadjuvant talazoparib for operable stage I breast cancer patients with a gBRCAm demonstrated marked activity. Although future studies are needed to validate these results, with this study it is the first time that a single-agent targeted therapy has shown a pathologic complete response (pCR) in women with BRCA mutations, including those with triple-negative breast cancer.

At this point, PARP-i offers a reasonable option of fairly well tolerated therapy to treat metastatic breast cancer in a chronic disease setting.

Conte: At present: advanced disease (the role as maintenance treatment after chemotherapy needs to be explored). Taking into account the mechanisms of acquired resistance to PARP-i, I am not so sure that these are ideal drugs in the adjuvant setting (maybe in combination with immune checkpoint inhibitors?).

Eniu: Future clinical trials will clarify this issue; perhaps even in prevention?

Saghatchian: Data are available for veliparib in the neoadjuvant setting, i.e. the phase III BrighTNess trial [7] of addition of veliparib plus carboplatin versus addition of carboplatin to standard neoadjuvant therapy for early-stage triple-negative breast cancer. In this trial, addition of veliparib to neoadjuvant carboplatin + paclitaxel followed by AC did not increase the pCR rate.

Due to the high chemosensitivity of tumors carrying BRCA mutation, it might prove difficult to show an additional effect of PARP-i in the neoadjuvant setting, with pCR as a study endpoint.

The ongoing phase III OlympiA trial holds much promise for high-risk BRCAm patients with olaparib in the adjuvant setting, administered during 12 months in addition to standard adjuvant treatment. 


\section{Participants}

Eleni Andreopoulou, M.D.

Weill Cornell Breast Center

Division of Hematology and Medical Oncology

Weill Cornell Medicine

425 East 61st Street, 8th Floor, New York, NY 10065, USA

ela9082@med.cornell.edu

Alexandru Eniu, MD, PhD

Department of Breast Tumors

Cancer Institute 'Ion Chiricuta'

Republicii 34-36, 400015 Cluj-Napoca, Romania

aleniu@iocn.ro

\section{References}

1 Robson M, Im SA, Senkus E, Xu B, Domchek SM, Masuda N, Delaloge S, Li W, Tung N, Armstrong A, Wu W, Goessl C, Runswick S, Conte P: Olaparib for metastatic breast cancer in patients with a germline BRCA mutation. N Engl J Med 2017;377:523-533. Erratum in: N Engl J Med 2017;377:1700.

2 Davies H, Glodzik D, Morganella S, et al: HRDetect is a predictor of BRCA1 and BRCA2 deficiency based on mutational signatures. Nat Med 2017;23:517-525.

3 Telli ML, Turner NC, Mailliez A, et al: Abstract P1-1403: ABRAZO: Exposure-efficacy and -safety analyse of breast cancer patients with germline BRCA1/2 mutations receiving talazoparib in a phase 2 open-label trial. Cancer Res 2018;78(suppl):P1-14-03-P1-14-03.
Prof. Pierfranco Conte

Division of Medical Oncology 2

Istituto Oncologico Veneto IRCCS

via Gattamelata 64, 35128 Padova, Italy

pierfranco.conte@unipd.it, pierfranco.conte@ioveneto.it

Dr. Mahasti Saghatchian, MD, MSc

Breast Cancer Unit

American Hospital of Paris

63, Bd Victor Hugo, 92200 Neuilly-sur-Seine, France

mahasti.saghatchian@ahparis.org
4 Litton J, Rugo HS, Ettl J, Hurvitz S, Gonçalves A, Lee K, Fehrenbacher L, Yerushalmi R, Mina LA, Martin M, Roché H, Im Y-H, Quek RGW, Tudor IC, Hannah AL, Eiermannand W, Blum JL: EMBRACA: a phase 3 trial comparing talazoparib, an oral PARP inhibitor, to physician's choice of therapy in patients with advanced breast cancer and a germline BRCA mutation (Abstract GS6-07). Proceedings of the 2017 San Antonio Breast Cancer Symposium; December 5-9, 2017; San Antonio, TX. Cancer Res 2018;78(suppl):abstr GS6-07. 5 Tutt A, Tovey H, Cheang MCU, et al: Carboplatin in BRCA1/2-mutated and triple-negative breast cancer BRCAness subgroups: the TNT Trial. Nat Med 2018; 24:628-637.
6 Gourd K: San Antonio Breast Cancer Symposium 2016. BROCADE Trial. Lancet Oncol 2017;18:176.

7 Loibl S, O'Shaughnessy J, Untch M, et al: Addition of the PARP inhibitor veliparib plus carboplatin or carboplatin alone to standard neoadjuvant chemotherapy in triple-negative breast cancer (BrighTNess): a randomised, phase 3 trial. Lancet Oncol 2018;19:497-509. 